\title{
Online asthma management for children is cost-effective
}

To the Editor:

eHealth interventions have been proposed as an appealing method to improve health outcomes and reduce healthcare costs [1-3]. However, the development of an eHealth intervention is associated with high costs and this investment needs to be balanced by increased clinical effectiveness and related cost savings. Unfortunately, solid evidence for the effectiveness of eHealth with regard to health improvement is still limited [2-5], as is evidence regarding cost-effectiveness. This causes uncertainty about the effectiveness of eHealth and constitutes a barrier towards successful implementation in daily practice [6, 7]. Thus, it is necessary to assess both effectiveness and cost-effectiveness to convince colleagues and policymakers of its added value.

We assessed the cost-effectiveness of online asthma care for children alongside a randomised controlled trial (RCT) to evaluate the effectiveness of this eHealth intervention on health improvement [8]. In this RCT, 210 asthmatic children were randomly allocated into two groups of asthma management. In the usual care (UC) group, care consisted of routine 4-monthly outpatient visits where asthma control was assessed with an asthma control test (ACT)/children's asthma control test (C-ACT) [9]. Alternatively, in the virtual asthma clinic (VAC) group, outpatient visits were reduced by $50 \%$ and asthma control was monitored online with monthly web-based ACT/C-ACT tests. In the VAC group, children had more symptom-free days per month and their ACT/C-ACT scores were more improved. As such, this RCT proved that routine outpatient visits can partly be replaced by online asthma management using the VAC.

We carried out an a priori defined cost-effectiveness analysis with the hypothesis that online management via the VAC is cost-effective. Standard cost questionnaires were completed by the parents every 4 months to assess costs from both a healthcare and a societal perspective. Healthcare (or direct) costs included all costs related to medical conditions, prescribed medication and intervention costs (including development of the VAC and estimated hosting and licence costs). Societal (or indirect) costs consisted of the loss of productivity, travel costs for any medical condition and parking expenses. The costs were based on the Dutch guideline for cost analyses [10] although if prices were not available other sources were used. Prices were converted to the 2014 level using the Dutch consumer price index [11].

Mean incremental costs were weighted against the mean incremental effects in terms of asthma-related quality of life (QoL) and asthma control. Uncertainty boundaries of $95 \%$ for the incremental cost-effectiveness ratio (ICER) were determined nonparametrically using bootstrap analyses. In this bootstrap simulation, 1000 random samples of cost-effect pairs were selected with replacement. Results from the simulation were presented graphically in a scatter plot in which each dot signifies the ICER of one iteration of the bootstrap stimulation. Two validated questionnaires were used to assess QoL at 0,8 and 16 months: the Paediatric Asthma Caregiver's Quality of Life Questionnaire (PACQLQ) to assess QoL in the caregivers of young children (6-12 years) and the Paediatric Asthma Quality of Life Questionnaire (PAQLQ) for teenagers (12-16 years) $[12,13]$. Asthma control was measured by ACT/C-ACT as described above for the RCT. Differences in cost were assessed with a nonparametric Mann-Whitney U-test. The changes from baseline in ACT/C-ACT and PACQLQ/PAQLQ were analysed using linear mixed models.

After 16 months, asthma control in young children (C-ACT) was higher in the VAC group with a mean (95\% CI) difference of $1.17(0.09-2.25)$ points $(p=0.03)$ [8]. In the group of teenagers, no significant difference in ACT score between treatment arms was observed with a mean (95\% CI) difference of 0.88 $(-0.41-2.16)$ points. In addition, a $50 \%$ reduction in the number of outpatient visits did not have any relevant influence on PACQLQ and PAQLQ results (data not presented; $\mathrm{p}=0.95$ and 0.84 , respectively).

@ERSpublications

Online asthma management in children can (partly) substitute routine outpatient visits and is costeffective http://ow.ly/oabk30f7Sre

Cite this article as: van den Wijngaart LS, Kievit W, Roukema J, et al. Online asthma management for children is cost-effective. Eur Respir J 2017; 50: 1701413 [https://doi.org/10.1183/13993003.01413-2017]. 
Both direct and indirect costs per patient were significantly lower in the VAC group than in the UC group (median VAC €726.52/median UC €875.86 ( $\mathrm{p}=0.01)$ and median VAC $€ 102.79 /$ median UC $€ 124.56$ $(\mathrm{p}=0.02)$, respectively). This resulted in lower total costs from a societal perspective (direct plus indirect costs) for VAC compared to UC (median VAC €889.77/median UC $€ 1081.47$ ( $p=0.014)$ ). There were no significant differences in the following healthcare and societal costs: GP consultations, paediatrician consultations, other specialists, physiotherapists, consultations by phone, Emergency Room visits, hospital admissions and loss of productivity (data not presented). Online asthma management was associated with lower costs because of fewer outpatient clinic visits (median VAC €237.09/median UC €385.15 ( $\mathrm{p}<0.000)$ ) and lower travel expenses (median VAC $€ 94.09 /$ median UC $€ 120.15(\mathrm{p}=0.003)$ ).

In young children, the VAC resulted in both improved health outcomes and cost savings in 99\% of the 1000 bootstrapped replications with a mean cost saving of $€ 352$ per patient (95th percentiles: $€ 67$ and $€ 652$ ) (figure 1a). In teenagers, considerable cost savings were realised in $90 \%$ of the bootstrapped replications with mean savings of $€ 852$ per patient (95th percentiles: $€-294$ and $€ 2584$ ) (figure 1b). Asthma control was similar in both groups and the percentage of bootstrapped replications that resulted in both improved health outcomes and cost savings was $74 \%$. Similar results were found for the cost-effectiveness planes concerning QoL and cost (figures 1c and 1d). QoL was maintained whereas cost savings were realised for online care in both age groups.

In summary, we have demonstrated the cost-effectiveness of online asthma management for children with a $50 \%$ reduction in the number of routine outpatient visits compared with UC. In young children, online care results in both improved health outcomes and substantial cost savings. In teenagers, UC and online care are equally effective with respect to clinical outcome but considerable cost-savings are realised with online care. This cost-effectiveness analysis supports implementation of online asthma management from
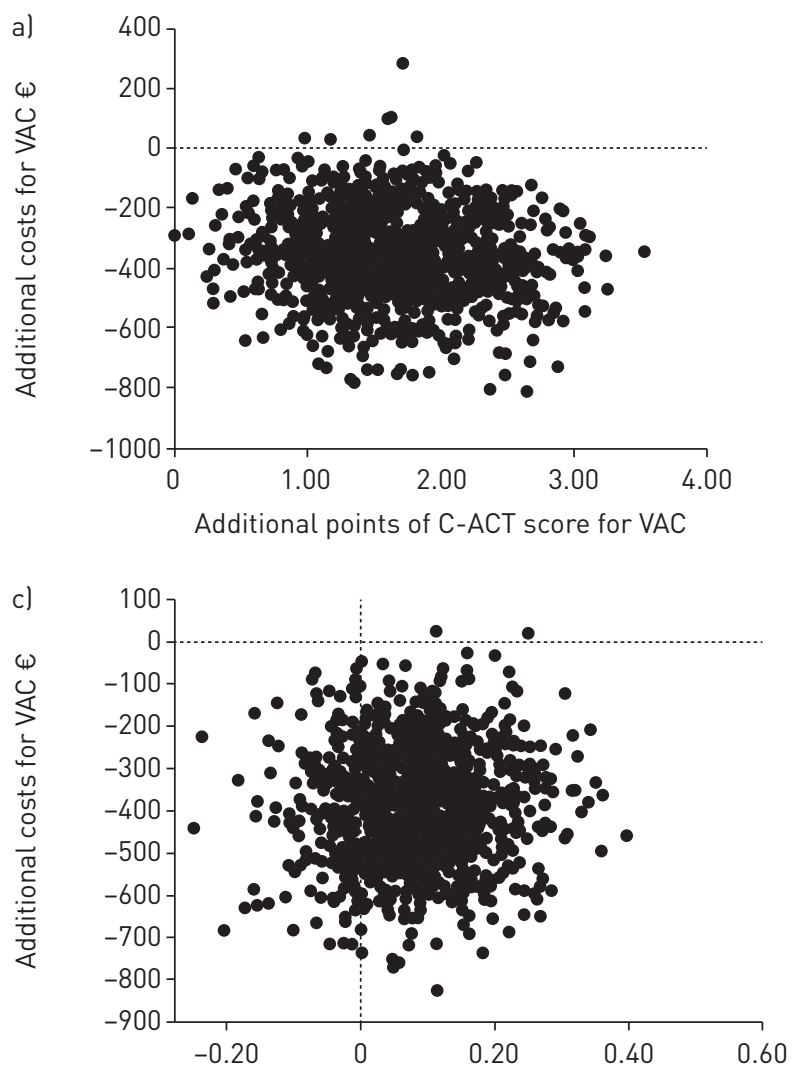

Additional effects on PACQLQ score for VAC
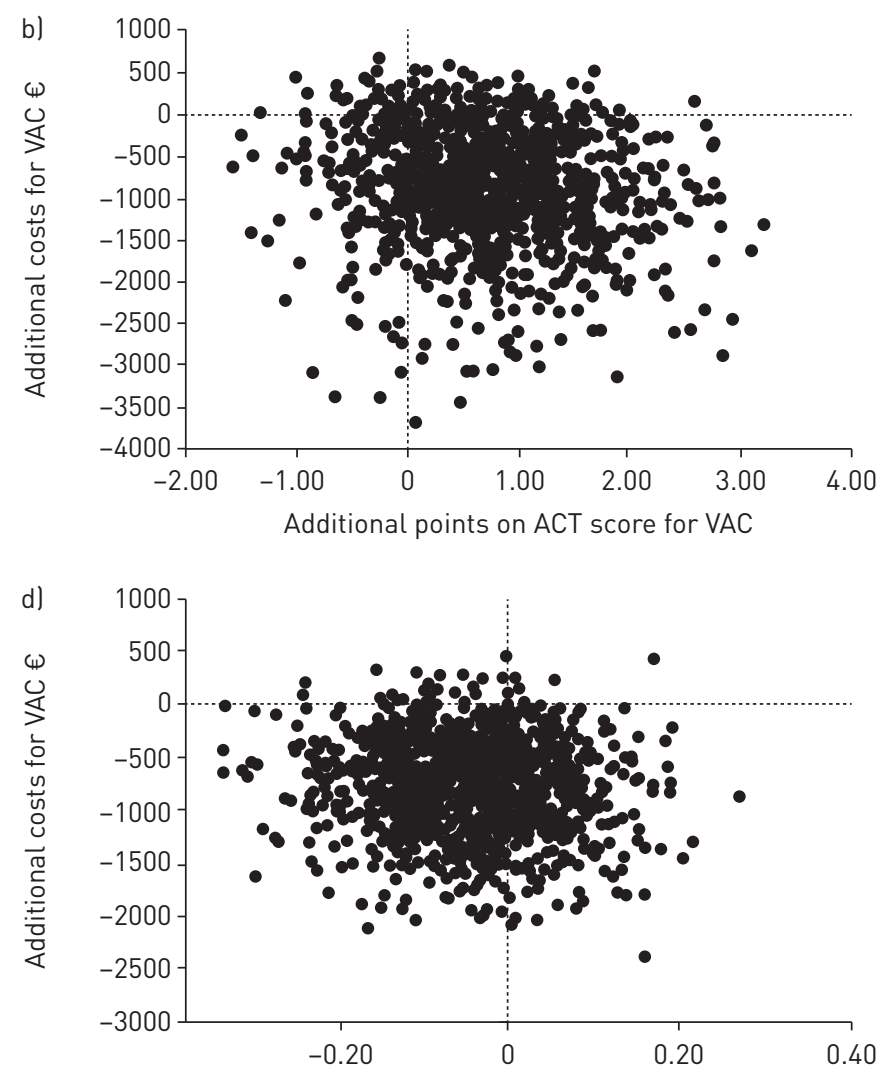

Additional effects on PAQLQ score for VAC

FIGURE 1 Cost-effectiveness planes showing the cost-effectiveness of a virtual asthma clinic (VAC) compared with usual care (1000 bootstrapped estimates). Dots represent the societal costs (direct plus indirect costs) plotted against asthma control for a) young children (6-11 years; asthma control measured by C-ACT) and b) teenagers (12-16 years; asthma control measured by ACT) or against quality of life for c) caregivers of young children (quality of life measured by PACQLQ) and d) teenagers (quality of life measured by PAQLQ). The South-East quadrants in a, b, and c indicate that the VAC dominates usual care (i.e. effectiveness is higher and costs are lower); however, the lower quadrants in $d$ indicate that while costs are lower, effectiveness is the same. C-ACT: children's asthma control test; ACT: asthma control test; PACQLQ: Paediatric Asthma Caregiver's Quality of Life Questionnaire; PAQLQ: Paediatric Asthma Quality of Life Questionnaire. 
a health economics perspective. While evidence on the clinical effectiveness of eHealth is accumulating, the evidence of its cost-effectiveness remains scarce $[3,14]$. The main limitations on the economic evaluation of eHealth are the lack of RCTs and the absence of quality data and appropriate measures [14, 15]. In addition, most eHealth interventions are used in addition to standard care, in contrast to our study where eHealth partly replaces UC. National implementation of the VAC has the potential to effect costs savings of $€ 10.7$ million per year (including both direct and indirect costs) based on the \pm 30000 children with asthma treated by paediatricians in The Netherlands. Online paediatric asthma care in other countries may result in substantial cost savings as well, especially when healthcare costs are higher or travel distances are larger. One could argue that the frequency of routine visits in real life differs from the fixed protocol in the RCT and that some patients should be seen less frequently and others more frequently. A general approach to individual patients is not desirable and the focus of healthcare is shifting from the volume of services delivered to added patient value. It is important to tailor healthcare to individual patient's wishes, in agreement with the doctor's needs. This is one of the strengths of the VAC: it reflects a development towards "personalised eHealth". Additionally, it is a nice example of value-based healthcare: achieving high value for patients with care adapted to the individual, while reducing costs by replacing infrequent traditional outpatient visits by more frequent online monitoring, which is both more efficient and more patient friendly.

We conclude that the VAC for children is an effective and cost-effective eHealth intervention to improve asthma care. Introducing this intervention as part of current asthma management is an attractive and very realistic option to optimise monitoring strategies in a more personalised way. The next step is implementation and routine use of this intervention in daily practice.

Lara S. van den Wijngaart ${ }^{1}$, Wietske Kievit ${ }^{2}$, Jolt Roukema ${ }^{1}$, Annemie L.M. Boehmer ${ }^{3}$, Marianne L. Brouwer ${ }^{4}$, Cindy A.C. Hugen ${ }^{1}$, Laetitia E.M. Niers ${ }^{5}$, Arwen J. Sprij ${ }^{6}$, Eleonora R.V.M. Rikkers-Mutsaerts ${ }^{7}$, Bart L. Rottier ${ }^{8}$, Chris M. Verhaak ${ }^{9}$, Mariëlle W. Pijnenburg ${ }^{10}$ and Peter J.F.M. Merkus ${ }^{1}$

${ }^{1}$ Dept of Paediatric Pulmonology, Radboud University Medical Centre, Amalia Children's Hospital, Nijmegen, The Netherlands. ${ }^{2}$ Radboud Institute for Health Sciences, Radboud University Medical Centre, Nijmegen, The Netherlands. ${ }^{3}$ Dept of Paediatrics, Maasstad Hospital, Rotterdam, The Netherlands. ${ }^{4}$ Dept of Paediatrics, Canisius Wilhelmina Hospital, Nijmegen, The Netherlands. ${ }^{5}$ Dept of Paediatrics, Maxima Medical Centre, Veldhoven, The Netherlands. ${ }^{6}$ Dept of Paediatric Pulmonology, Haga Hospital, Juliana Children's hospital, Den Hague, The Netherlands. ${ }^{7}$ Dept of Paediatric Pulmonology, Leiden University Medical Centre, Leiden, The Netherlands. ${ }^{8}$ Dept of Paediatric Pulmonology and Allergology, Groningen Research Institute for Asthma and COPD, University of Groningen, University Medical Centre Groningen, Beatrix Children's Hospital, Groningen, The Netherlands. ${ }^{9}$ Dept of Psychology, Radboud University Medical Centre, Nijmegen, The Netherlands. ${ }^{10}$ Dept of Paediatric Pulmonology, Erasmus Medical Centre, Sophia Children's Hospital, Rotterdam, The Netherlands.

Correspondence: L.S. van den Wijngaart, Radboud University Medical Centre, Amalia Children's Hospital, PO 9101, 6500 HB Nijmegen, The Netherlands. E-mail: lara.vandenwijngaart@radboudumc.nl

Received: July 132017 | Accepted: July 232017

Support statement: This study was funded by the Netherlands Lung Foundation (Grant: 3.4.10.016) and the Innovation Foundation of the Dutch Health Insurance Companies (Grant: B 10-135). The funders had no role in trial design, data collection, analysis or reporting. Funding information for this article has been deposited with the Crossref Funder Registry.

Conflict of interest: Disclosures can be found alongside this article at erj.ersjournals.com

Acknowledgements

Author roles: Merkus, Peter; Pijnenburg, Mariëlle; Verhaak, Chris designed the study and obtained funding. van den Wijngaart, Lara; Roukema, Jolt; Boehmer, Annemie; Brouwer, Marianne; Verhaak, Chris; Niers, Laetitia; Sprij, Arwen; Rikkers-Mutsaerts, Eleonora; Rottier, Bart; Pijnenburg, Mariëlle; Merkus, Peter all participated as investigators, recruited patients and performed treatments. van den Wijngaart, Lara carried out the analyses. Kievit, Wietske conducted the cost-effectiveness analysis. van den Wijngaart, Lara; Kievit, Wietske; Roukema, Jolt; Merkus, Peter operated as the writing committee and drafted the manuscript until it reached its final form. All authors commented on the manuscript and its revisions. Merkus, Peter is the guarantor of the study. Ethical approval: This study was approved by all local ethics committees. All participants provided written informed consent. Data sharing: Patient-level data are available on reasonable request from the corresponding author. Patient consent was not obtained but the data presented are anonymised and risk of identification is absent. Transparency declaration: The lead author affirms that this manuscript is an honest, accurate and transparent account of the study being reported, that no important aspects of the study have been omitted, and that any discrepancies from the study as planned (and, if relevant, registered) have been explained. Prior presentations: Results of this randomised controlled trial were presented at the Congress of the European Respiratory Society (Amsterdam, 2015). Results of the cost-effectiveness analysis were presented at the Congress of International Paediatric Pulmonology (Naples, 2016). Thanks: We are grateful to all the participating children and parents for their contribution to this study, as well as collaborating paediatricians and Sintnicolaas, Coosje (Radboud University Medical Centre, Amalia Children's Hospital, Department of Paediatric Pulmonology, Nijmegen, The Netherlands); Stevens, Petra (Canisius Wilhelmina Hospital, Department of Paediatrics, Nijmegen, The Netherlands); Verwaal, Mariël (Maasstad Hospital, Department of Paediatrics, Rotterdam, The Netherlands); Bolder, Carola (Maxima 
Medical Centre, Department of Paediatrics, Veldhoven, The Netherlands); Winters, Ans (Leiden University Medical Centre, Department of Paediatric Pulmonology, Leiden, The Netherlands); Mulder, Ineke and van Baak, Christine (both Haga Hospital, Juliana Children's Hospital, Department of Paediatric Pulmonology, The Hague, The Netherlands); Spierings, Magda and Hiddinga, Jantine (both Erasmus Medical Centre, Sophia Children's Hospital and Kinderhaven/ Havenziekenhuis, Department of Paediatric Pulmonology, Rotterdam, The Netherlands) for their enthusiasm and their efforts in recruiting and monitoring the patients. We acknowledge Hop, Wim (Erasmus Medical Centre, Department of Biostatistics, Rotterdam, The Netherlands) and Moll, Henriëtte (Erasmus Medical Centre, Sophia Children's Hospital, Department of Paediatrics, Rotterdam, The Netherlands) for performing the interim safety analysis and also commemorate van Gent, Rene (Maxima Medical Centre, Department of Paediatrics, Veldhoven, The Netherlands) and thank him for his contributions to this study.

\section{References}

1 McLean S, Chandler D, Nurmatov U, et al. Telehealthcare for asthma: a Cochrane review. CMAJ 2011; 183: E733-E742.

2 Zhao J, Zhai YK, Zhu WJ, et al. Effectiveness of telemedicine for controlling asthma symptoms: a systematic review and meta-analysis. Telemed J E Health 2015; 21: 484-492.

3 Ekeland AG, Bowes A, Flottorp S. Effectiveness of telemedicine: a systematic review of reviews. Int J Med Inform 2010; 79: 736-771.

$4 \quad$ McLean S, Protti D, Sheikh A. Telehealthcare for long term conditions. BMJ 2011; 342: d120.

5 McLean S, Sheikh A, Cresswell K, et al. The impact of telehealthcare on the quality and safety of care: a systematic overview. PloS one 2013; 8: e71238.

6 Bergmo TS. How to measure costs and benefits of eHealth interventions: an overview of methods and frameworks. J Med Internet Res 2015; 17: e254.

7 Miller EA. Solving the disjuncture between research and practice: telehealth trends in the 21st century. Health Policy 2007; 82: 133-141.

8 van den Wijngaart LS, Kievit W, Roukema J, et al. A virtual asthma clinic for children: fewer routine outpatient visits, same asthma control. Eur Respir J 2017; 50: 1700471.

9 Juniper EF, O'Byrne PM, Guyatt GH, et al. Development and validation of a questionnaire to measure asthma control. Eur Respir J 1999; 14: 902-907.

10 Oostenbrink JB, Koopmanschap MA, Rutten FFH. Manual for costing: methods and standard costs for economic evaluations in health care (in Dutch). Amstelveen, Dutch Health Insurance Executive Board, 2011.

11 Statistics Netherlands. Consumer price index 2014. www.cbs.nl Date last accessed: September 14, 2017.

12 Juniper EF, Guyatt GH, Feeny DH, et al. Measuring quality of life in the parents of children with asthma. Qual Life Res 1996; 5: 27-34.

13 Juniper EF, Guyatt GH, Feeny DH, et al. Measuring quality of life in children with asthma. Qual Life Res 1996; 5: 35-46.

14 Mistry H. Systematic review of studies of the cost-effectiveness of telemedicine and telecare. Changes in the economic evidence over twenty years. J Telemed Telecare 2012; 18: 1-6.

15 de la Torre-Diez I, Lopez-Coronado M, Vaca C, et al. Cost-utility and cost-effectiveness studies of telemedicine, electronic, and mobile health systems in the literature: a systematic review. Telemed J E Health 2015; 21: 81-85. 